\title{
Influence of buffer layers on Ni thin film structure and graphene growth by CVD
}

\author{
Elif Ozceri ${ }^{1}$ and Yusuf Selamet ${ }^{2}$ \\ ${ }^{1}$ Department of Materials Science and Engineering, Izmir Institute of Technology, Urla Izmir 35430, \\ Turkey \\ ${ }^{2}$ Department of Physics, Izmir Institute of Technology, Urla Izmir 35430, Turkey \\ E-mail: elifozceri@iyte.edu.tr
}

Received 11 May 2015, revised 10 August 2015

Accepted for publication 25 August 2015

Published 9 October 2015

\begin{abstract}
Buffer and/or adhesive layers were used to decrease the dewetting of Ni thin film at graphene growth temperatures of around $900{ }^{\circ} \mathrm{C}$. Depositing a thin buffer $\left(\mathrm{Al}_{2} \mathrm{O}_{3}\right)$ layer onto $\mathrm{SiO}_{2} / \mathrm{Si}$ substrate significantly reduced the dewetting effect and surface roughness of Ni catalyst film. Thin adhesive $(\mathrm{Cr})$ layers with or without $\mathrm{Al}_{2} \mathrm{O}_{3}$ buffer layers increased the texturing in (1 111$)$ orientation, which was promoted by growing at an elevated temperature $\left(450{ }^{\circ} \mathrm{C}\right)$. The effects of pretreatment and growth temperature on crystal orientation, grain size and surface roughness of Ni film were analyzed. Our results indicated a large positive correlation coefficient between the film thickness and surface roughness for thinner and non-buffered films, and a negative correlation coefficient between the thickness and $900{ }^{\circ} \mathrm{C}$-annealed film roughness for thicker and buffered films. The graphene coverage was greatly improved over the films grown with $\mathrm{Al}_{2} \mathrm{O}_{3}$ and/or $\mathrm{Cr}$ layers. In summary, we suggest that growing high quality, large area, 1- or 2-layer graphene on polycrystalline $\mathrm{Ni}$ transition metal thin film is optimized by using $\mathrm{Al}_{2} \mathrm{O}_{3}$ and/or Cr layers to reduce Ni dewetting, surface roughness, and groove depth while controlling grain size and texturing in (111) orientation by annealing at $900{ }^{\circ} \mathrm{C}$.
\end{abstract}

Keywords: thin film dewetting, buffered growth, film pretreatment, CVD, graphene growth

S Online supplementary data available from stacks.iop.org/JPhysD/48/455302/mmedia

(Some figures may appear in colour only in the online journal)

\section{Introduction}

To obtain high-quality graphene, substantial efforts have been directed towards optimizing the chemical vapour deposition (CVD) conditions [1-3]; however, thin film, serving as both catalyst and support [4], has a pronounced effect on graphene growth, perhaps even overshadowing graphene growth conditions.

Compared to other widely used materials such as $\mathrm{Cu}$, $\mathrm{Pt}$, and Ir, graphitic layers grown on $\mathrm{Ni}$ substrate have been reported to possess a higher degree of crystallinity [5]. Additionally, particular studies have reported that graphene can easily grow on an (111) oriented Ni surface due to a reasonable lattice match [6-9] and to the lower energy existing at the interface than other crystallographic orientations $[10,11]$. (It should be noted, however, that at least one study reports that local binding variations can occur between graphene and the lattice-mismatched underlying metal, and yet not affect continuous graphene growth [12].) A further point of consideration is that Ni has higher dehydrogenation and carbon solubility at high temperatures than many other materials [13].

On a polycrystalline $\mathrm{Ni}$ surface, grain boundaries can interfere with carbon segregation, forming grooves with higher carbon concentration than for a less granular surface $[3,8$, 14]. Multilayer graphene grows in these grooves, reducing precipitation uniformity $[13,15]$. Thus, grain boundaries on polycrystalline Ni film can be detrimental to the mechanical and electrical properties of graphene [16, 17] and limit the graphene flake size $[11,18,19]$.

Presenting a further complication, $\mathrm{Ni}$ grains tend to form clusters, and exposing the substrate surface to extreme temperatures results in Ni film dewetting. This effect is due to 
internal forces in the film being larger than those with a film and substrate, where excess energy is supplied by pretreatment [20-22] and the effect becomes more prominent (and increases surface roughness) with increasing temperature. To lessen the consequences of dewetting, thicker $(>400 \mathrm{~nm}) \mathrm{Ni}$ films have been used for graphene growth [23]. However, thicker films lead to more carbon solution in the film, which in turn leads to multilayer graphene growth. As an alternate counter measure, a buffer layer can be used to decrease the dewetting effect and reduce surface roughness; $\mathrm{Al}_{2} \mathrm{O}_{3}$ has been widely used as a buffer layer on top of $\mathrm{SiO}_{2}$ in the CVD growth of CNT and graphene [24-27]. $\mathrm{Al}_{2} \mathrm{O}_{3}$ has high thermal conductivity and generally functions better than $\mathrm{SiO}_{2}$ as a support layer for the hydrocarbon CVD process [28]. Additionally, $\mathrm{Cr}$ is a commonly used material that has a high oxidation tendency and increases the adherence of metal thin films onto substrate surfaces [19]. When used in conjunction with a metal, the $\mathrm{Al}_{2} \mathrm{O}_{3}$ buffer prevents metal silicide formation between the substrate and the catalyst metal particles [29]. Moreover, metal on an oxide buffer layer exhibits much less mobility [30] due to increased adhesion [31] between the film and the substrate. Hence, to grow high-quality, large-area, and 1-2-layer graphene on polycrystalline Ni transition metal (TM) thin film is of fundamental importance to reduce $\mathrm{Ni}$ dewetting and the surface roughness and groove depth of $\mathrm{Ni}$ film, and to have the largest grain sizes and texturing in (1 111$)$ orientation.

In this work, we investigated the importance of optimizing thin film properties for the CVD growth of graphene. Thin film properties, and hence graphene growth, were greatly enhanced by using a buffer $\left(\mathrm{Al}_{2} \mathrm{O}_{3}\right)$ and/or an adhesion layer (Cr). We demonstrated that reduced $\mathrm{Ni}$ dewetting and surface roughness and texturing in (111) orientation provided highquality, large-area graphene of 1-2 layers on polycrystalline Ni TM thin film.

\section{Experimental details}

$\mathrm{Ni}$ films with $\mathrm{Al}_{2} \mathrm{O}_{3}$ and/or thin $\mathrm{Cr}$ layers were deposited on $\mathrm{SiO}_{2} / \mathrm{Si}$ wafers by magnetron sputtering. Cleaning and preparing the substrate prior to thin film deposition and graphene growth were carried out using an ultrasonic bath (details are given in the online supporting information) (stacks.iop.org/ JPhysD/48/455302/mmedia). The growth rates for various deposition powers were determined from the growth time and film thickness, measured both by cross-sectional SEM and a surface profiler. Ni films with and without an $\mathrm{Al}_{2} \mathrm{O}_{3}$ and/or $\mathrm{Cr}$ buffer and adhesive layers were deposited over $\mathrm{SiO}_{2}(\mu \mathrm{m}) /$ $\mathrm{Si}$ and $\mathrm{SiO}_{2}(230 \mathrm{~nm}) / \mathrm{Si}$ substrates. Some samples were grown with substrate heating, which was accomplished using halogen heaters behind the substrate. All the films were grown under $9.95 \mathrm{sccm}$ Ar flow.

For grain enlargement, the films were annealed at 800, 900, and $950{ }^{\circ} \mathrm{C}$ under $150 \mathrm{sccm}$ Ar flow environmental pressure for $60 \mathrm{~min}$. All the as-grown and annealed films were characterized by XRD, AFM, SEM, and surface profilometry. The XRD patterns were collected with a Philips X'Pert diffractometer with $\mathrm{Cu} \mathrm{K} \alpha$ radiation and analysed with $\mathrm{X}$ 'pert
HighScore Peak Search software. The crystal qualities were analysed by the peak intensity ratios from XRD $\theta-2 \theta$ measurements and grain sizes were calculated using Scherer's formula. The surface roughness determinations of the films were obtained from AFM surface topography measurements. SEM was used for topography and thickness measurements.

To grow graphene over films with various thicknesses, numerous growth parameters were applied using atmospheric pressure CVD (see the online supporting information) (stacks. iop.org/JPhysD/48/455302/mmedia). $\mathrm{Ar}$ and/or $\mathrm{H}_{2}$ were introduced for 20 or $50 \mathrm{~min}$ before introducing $\mathrm{CH}_{4}$. A 20 or $50 \mathrm{~min}$ pretreatment process with respective 40 or $10 \mathrm{~min}$ carbon dissolution times was used. The growths were also carried out at 800,900 , and $950{ }^{\circ} \mathrm{C}$. The heating rate was 28 ${ }^{\circ} \mathrm{C} \mathrm{min}^{-1}$. The CVD process was terminated by stopping the $\mathrm{CH}_{4}$ or $\mathrm{CH}_{4}: \mathrm{H}_{2}$ flow and the temperature of the chamber was cooled at $1,2,3,4,5,10$, or $15^{\circ} \mathrm{C} \mathrm{min}-1$.

Raman spectroscopy was used to identify the number of graphene layers on the Ni films as they were grown, without transferring to another substrate. An Ar-ion laser operating at $514.5 \mathrm{~nm}(2.41 \mathrm{eV})$ with a 600 groove $\mathrm{mm}^{-1}$ grating was used to obtain $\mathrm{D}, \mathrm{G}$, and $\mathrm{G}^{\prime}$ bands.

\section{Results and discussion}

\subsection{Scope of work}

In this study, eighteen films (referenced as Ni1 through Ni18) with four different structures were grown and characterized (figure 1(a)). Initially, Ni films of various thicknesses were grown on $\mathrm{SiO}_{2} / \mathrm{Si}$ substrates. Later, buffer layers including $\mathrm{Al}_{2} \mathrm{O}_{3}, \mathrm{Cr}$, and $\mathrm{Cr} / \mathrm{Al}_{2} \mathrm{O}_{3}$ were grown prior to the $\mathrm{Ni}$ film growth to improve the adherence of the films to the substrate at elevated temperatures. The growth parameters for the films are summarized in figure 1(b), along with the film thickness.

\subsection{Temperature}

It has been reported that the number of layers and the size of CVD-grown graphene are limited by the grain size of crystalline Ni films obtained after thermal annealing [8, 32]. This theory was tested in the initial part of the study, during which $\mathrm{Ni}$ films grown on $\mathrm{SiO}_{2} / \mathrm{Si}$ substrates were studied. These films were also used later on to show the effect of the buffer layers. XRD $\theta-2 \theta$ scans of the as-grown, 800 , and $900{ }^{\circ} \mathrm{C}$-annealed $\mathrm{Ni}$ films indicated that increasing the pretreatment temperature favoured texture in (1 111 ) orientation (figure 2). Furthermore, the average crystallite sizes were increased with the increasing pretreatment temperature (figure 2 inset). Annealing at $800^{\circ} \mathrm{C}$ was not sufficient to effectively disassociate the $\mathrm{CH}_{4}$ to obtain enough carbon and drive it into the film to sustain graphene growth. Therefore, graphene growth was carried out at $900{ }^{\circ} \mathrm{C}$ or higher.

It is generally accepted that during pretreatment, thin film tends to decrease its excess energy by minimizing the interfacial surface area between itself and the substrate [33]. The surface roughness increases and parts of the substrate might 
(a)

$1,2,3,4,5,6$, $9^{*}, 10^{*}, 13,14$

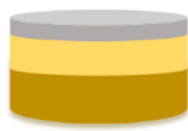

$7,8,11,12$

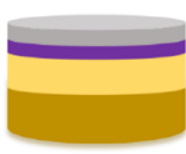

$16,18^{*}$

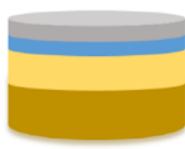

$15,17^{*}$

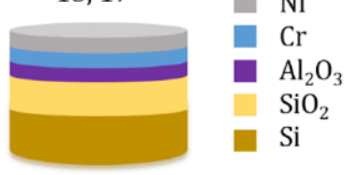

(b)

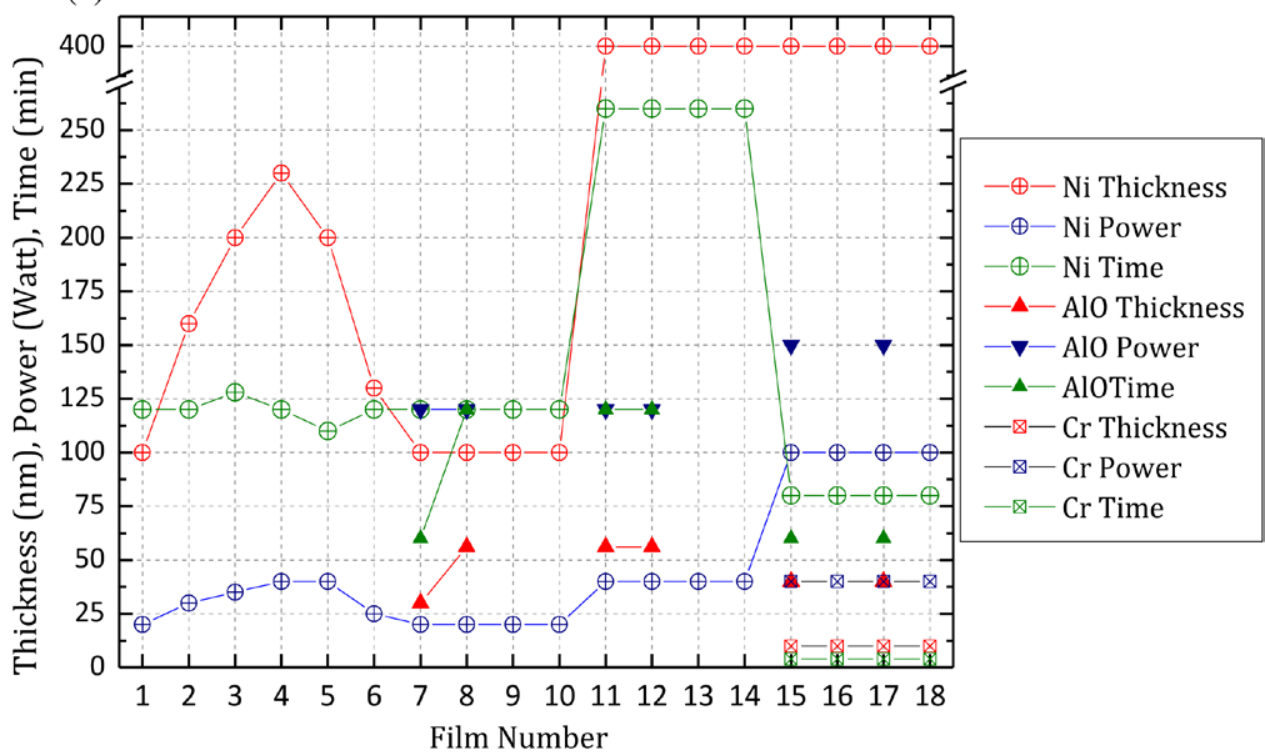

Figure 1. (a) Layer structures of Ni films with the sample numbers deposited on $\left.\mathrm{SiO}_{2} / \mathrm{Si} ;{ }^{*}\right)$ indicates that these films were deposited with substrate heating at $450^{\circ} \mathrm{C}$. (b) Composite plot of the dc/RF power, deposition time, and thickness of layers.

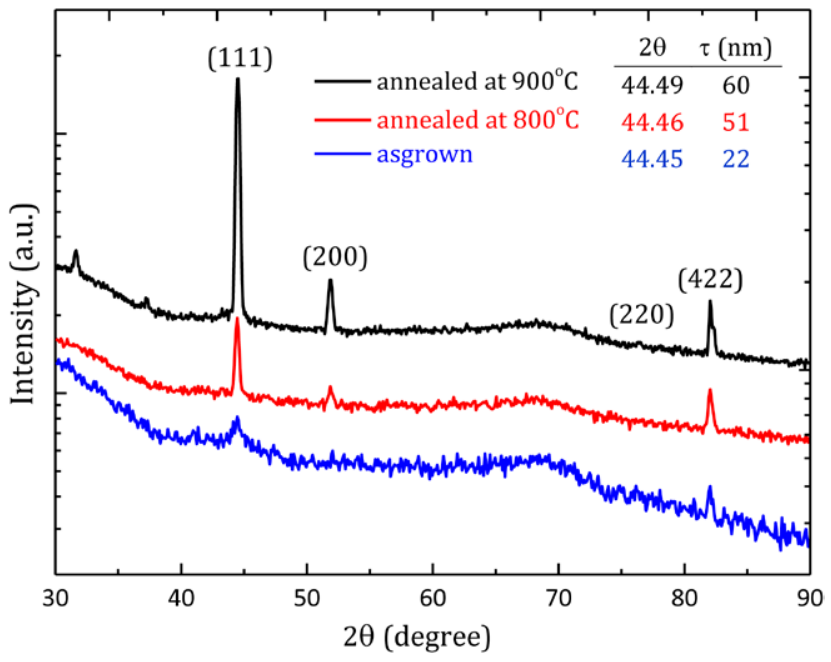

Figure 2. XRD $\theta-2 \theta$ scans of Ni1 samples. $2 \theta$ positions and average grain size values of the as-grown (blue), $800{ }^{\circ} \mathrm{C}$ (red), and $900{ }^{\circ} \mathrm{C}$ (black) annealed samples are given inside the plot.

be exposed, depending on the film thickness and degree of dewetting. The granular structure of the films and the effects of annealing were analysed with SEM and AFM. Figure 3 shows SEM and AFM topographic images of a $230 \mathrm{~nm}$ thick Ni4 film ((a) and (b)) and a $400 \mathrm{~nm}$ thick Ni13 film ((c) and (d)) without buffer layers or with a $\mathrm{Al}_{2} \mathrm{O}_{3}$ buffer layer (Ni11 (e) and (f)), annealed at $900{ }^{\circ} \mathrm{C}$. The surface roughness values are shown in figure 5. As can be clearly seen, the smoothest and least dewetted film is Ni11 with an $\mathrm{Al}_{2} \mathrm{O}_{3}$ buffer layer (the average rms value of Ni11 is about $38.8 \mathrm{~nm}$; Ni13 is about $72.9 \mathrm{~nm}$ ). Dewetting also increased with the pretreatment temperature. (Note: to achieve a continuous film and texture in (111) orientation at temperatures of $900{ }^{\circ} \mathrm{C}$ or above, $\mathrm{Al}_{2} \mathrm{O}_{3}$ buffer layers and/or $\mathrm{Cr}$ adhesive layers were deposited prior to the Ni film growth, as discussed below.)

\subsection{Structure}

Both substrate and buffer layers have characteristic peaks very close to the $2 \theta$ peak position of $\mathrm{Ni}$ (111). A simple calculation involving the atomic structure factor based on the FCC structure of Ni films yields a (111) peak position at $2 \theta=44.49^{\circ}$ (see the online supporting information) (stacks.iop.org/ JPhysD/48/455302/mmedia). Thermally grown $\mathrm{SiO}_{2}$ is usually amorphous and has a broad peak centred at $43^{\circ}$. The $\mathrm{NiO}$ peak is at $43.3^{\circ}$, the $\mathrm{Al}_{2} \mathrm{O}_{3}$ peak is at $43.9^{\circ}$, and the $\mathrm{Cr}$ peak is at $44.2^{\circ}$. In analysing the XRD $\theta-2 \theta$ measurement results of all the as-grown and annealed $\mathrm{Ni}$ films to calculate the grain sizes, the peaks appearing at this position were carefully fitted by considering all possible contributions from the substrate, buffer-adhesive layers, and $\mathrm{NiO}$ (figure 4).

All the as-grown Ni films were polycrystalline. The as-grown Ni1-6 films were grown along the (1 111$)$ direction and a well-defined (1 111$)$ peak appeared at $44.50^{\circ}$. 
(a)

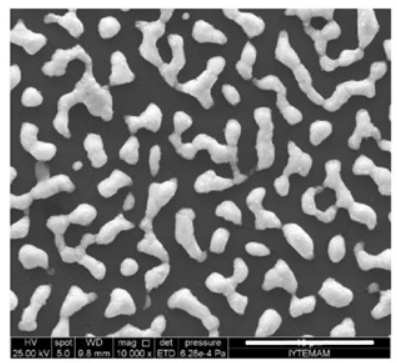

(c)

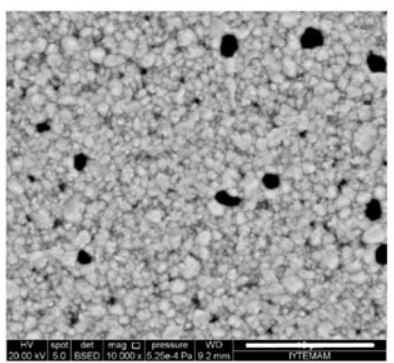

(e)

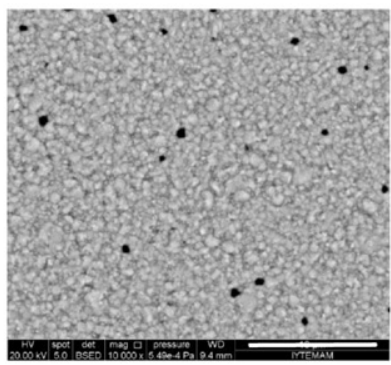

(b)

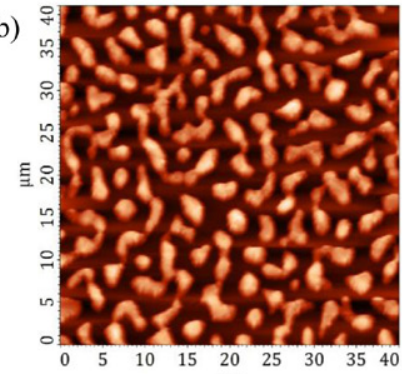

$\mu \mathrm{m}$

(d)

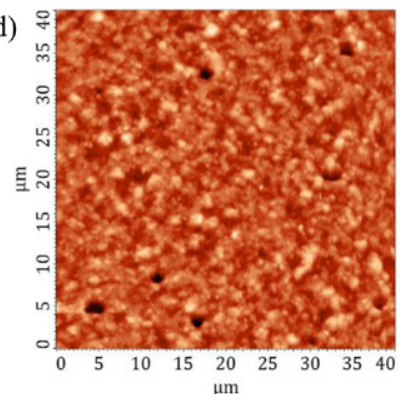

(f)

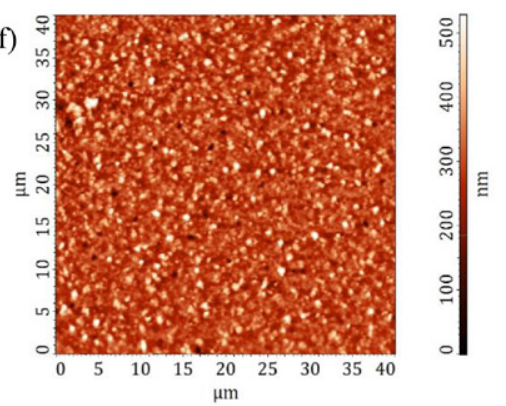

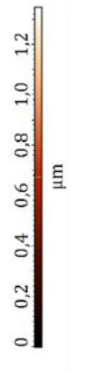

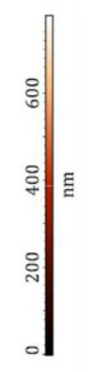

Figure 3. SEM micrographs (a), (c), and (e) and AFM topographic images (b), (d), and (f) of Ni4 (a) and (b), Ni13 (c) and (d) and Ni11 (e and f) films annealed at $900{ }^{\circ} \mathrm{C}$. The scale bar on the SEM images indicates $10 \mu \mathrm{m}$.

Among these layers, the film grown with the lowest power and least thickness had the greatest texturing in the (1 111$)$ orientation.

The Ni7 film was grown with a $30 \mathrm{~nm} \mathrm{Al}_{2} \mathrm{O}_{3}$ buffer layer and the film 8 was grown with a $60 \mathrm{~nm} \mathrm{Al}_{2} \mathrm{O}_{3}$ buffer layer. The intensity ratios of the $\mathrm{Ni}(1111)$ peak to other orientation were higher in the XRD measurements of the annealed Ni7 film compared to those of the Ni8 film. This was an indication of the thinner buffer more effectively favouring the (111) orientation of the Ni film (see the online supporting information) (stacks.iop.org/JPhysD/48/455302/mmedia).

To study the effects of Ni film thickness on crystal orientation, $400 \mathrm{~nm}$ thick layers (Ni11-14) were grown. All four were grown on a $\mathrm{SiO}_{2} / \mathrm{Si}$ substrate; Ni11 and Ni12 also had an $\mathrm{Al}_{2} \mathrm{O}_{3}$ buffer layer. The $\mathrm{SiO}_{2}$ layer thickness was $1 \mu \mathrm{m}$ for $\mathrm{Ni11}$ and Ni13 and 230 nm for Ni12 and Ni14. Annealing these samples increased texturing in the (220) direction for all four samples. There was a large strain in the as-grown samples, as indicated by the upshifts of the XRD peak positions of the samples with the annealing due to strain relief (see the online supporting information figure S2) (stacks.iop.org/ JPhysD/48/455302/mmedia). The strain was $52 \%$ and $44 \%$ for Ni11 and Ni13, respectively, and $38 \%$ and $31 \%$ for $\mathrm{Ni12}$ and Ni 14, respectively. The buffer layer of Ni11 and Ni12 apparently further contributed to strain relief.

\subsection{Substrate heating}

Some studies have reported an increase in texturing in the (111) orientation by heating the substrate during thin film deposition [34]. To study the effects of substrate heating on texture, two $100 \mathrm{~nm}$ thick Ni films (Ni9 and Ni10) were grown at a substrate temperature of $450{ }^{\circ} \mathrm{C}$. These films, however, exhibited dominant texturing in the (200) orientation (see the online supporting information) (stacks.iop.org/ JPhysD/48/455302/mmedia).

Ni17 and Ni18 were also deposited by substrate heating at $450{ }^{\circ} \mathrm{C}$. $\mathrm{Ni} 17$ had $\mathrm{Cr} / \mathrm{Al}_{2} \mathrm{O}_{3}$ and $\mathrm{Ni} 18$ had a $\mathrm{Cr}$ buffer over the $\mathrm{SiO}_{2} / \mathrm{Si}$ substrate. Similar to Ni9-10, these as-grown films were predominantly oriented in the (200) direction as compared to the samples deposited under the same conditions but without substrate heating. Nevertheless, the non-buffered Ni9 and Ni10 films had lower intensity (111) peaks in contrast to the Ni17 and Ni18 films. Therefore, it can be concluded that the $\mathrm{Cr}$ buffer caused the Ni film to have (111) preferentially oriented grains. Moreover, when annealing on the samples with a Cr buffer was performed for $60 \mathrm{~min}$, the intensity ratio of (111)/(200) further increased. When the Ni7, Ni8, Ni11, and Ni12 films were compared with the Ni15-18 films, it was seen that the contribution of the $\mathrm{Cr}$ layer was more than that of the $\mathrm{Al}_{2} \mathrm{O}_{3}$ buffer layer in texturing $\mathrm{Ni}$ film in the (1 111 ) orientation. 


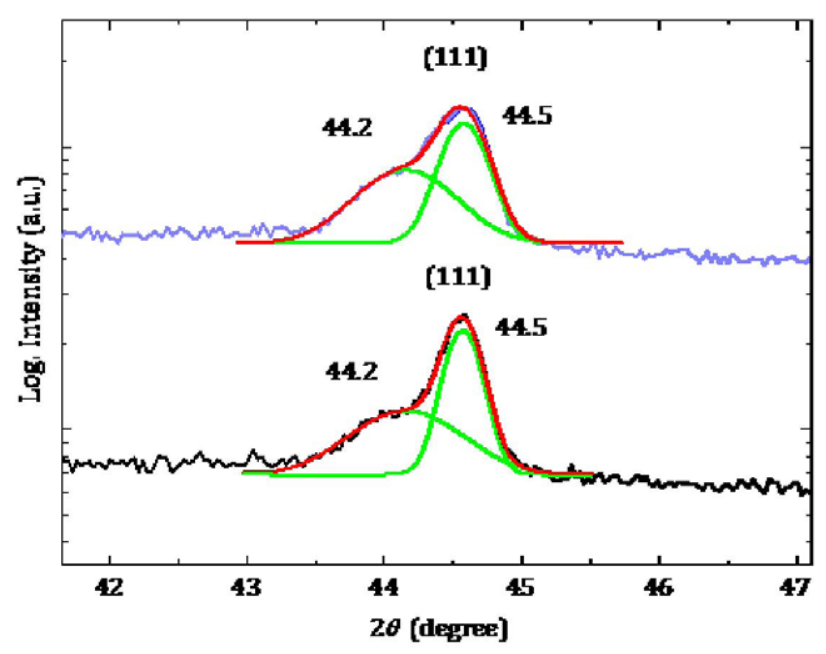

(a)

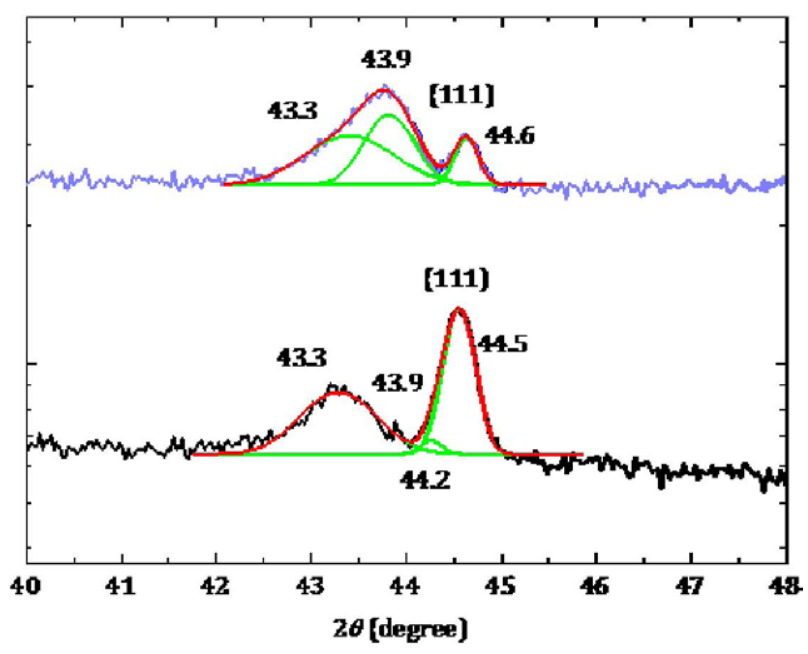

(b)

Figure 4. Fitted XRD $\theta-2 \theta$ measurements of Ni18 (a) and Ni16 (b) films. The black line indicates the as-grown and the light blue line indicates annealing at $900{ }^{\circ} \mathrm{C}$ for $60 \mathrm{~min}$.

The average grain sizes of the crystallites in the (111) orientation were calculated by using Scherer's formula. The average grain sizes for all the as-grown samples were roughly the same. The grain sizes increased with increasing the annealing temperature (see the online supporting information figure S4) (stacks.iop.org/JPhysD/48/455302/mmedia).

\subsection{Surface roughness}

The surface roughness (root mean square, RMS values) of both the as-grown and annealed Ni films was obtained by averaging several AFM topography images for each sample. Figure 5 shows the comparison of the as-grown thickness to the as-grown roughness and roughness after $900{ }^{\circ} \mathrm{C}$ annealing of all the Ni films studied here. The film thickness was obtained by both surface profilometry and cross-sectional SEM. The thickness measurements obtained by both techniques were in very good agreement. A striking similarity between the thickness and as-grown roughness measurements was observed, even though they were obtained with different techniques. There was a large positive correlation between the thickness and as-grown film roughness (Pearson correlation coefficient of 0.671 ), particularly for Ni films $1-8$, for which the positive correlation coefficient between the thickness and as-grown roughness RMS values was 0.982 , and between the thickness and annealed film roughness was 0.884 . On the other hand, there was a negative correlation coefficient between the thickness and annealed $\left(900^{\circ} \mathrm{C}\right)$ film roughness of -0.651 for films $9-18$. That is, as the film thickness increased the annealed film roughness decreased even though the as-grown roughness increased. Therefore, it can be said that the annealed film roughness was inversely proportional to the thickness for the thicker film numbers 12-18 (see the online supporting information) (stacks.iop.org/JPhysD/48/455302/mmedia).

Figure 5 also clearly shows a contribution from the buffer layer and thickness on the $\mathrm{Ni}$ film properties. Upon inspecting the annealed samples with lower roughness RMS values, it was evident that those with an $\mathrm{Al}_{2} \mathrm{O}_{3}$ buffer ( $\mathrm{Ni7}$, Ni8, Ni11, and Ni12 films) had a smoother surface compared to those without an $\mathrm{Al}_{2} \mathrm{O}_{3}$ buffer (Ni films of 1-6, 13 and 14). (The Ni9 and Ni10 films were grown with substrate heating, and thus are not included in the discussion.)

No significant difference was observed between the asgrown roughness RMS values of thicker (about $400 \mathrm{~nm}$ ) Ni films $(11,12,13$, and 14). However, when the Ni film thickness and roughness RMS values of the annealed samples were compared, it was evident that the thicker Ni films (13 and 14) had almost two times higher roughness than those with $\mathrm{Al}_{2} \mathrm{O}_{3}$ buffer layers (11 and 12). In addition, increasing the $\mathrm{Ni}$ film thickness resulted in bigger Ni particles on the surface (figure 3), whereas the average crystallite size did not change significantly (see the online supporting information figure S4) (stacks.iop.org/JPhysD/48/455302/mmedia). Hence, it can be concluded that the $\mathrm{Al}_{2} \mathrm{O}_{3}$ buffer was very effective in reducing the roughness of the Ni films at high temperatures (figure 5).

Sputtering power also had a strong influence on the asgrown film roughness. Films grown with a higher power but shorter time for a thickness of $400 \mathrm{~nm}$ (Ni15 through Ni18) had a substantially increased surface roughness (figure 5). The $\mathrm{Al}_{2} \mathrm{O}_{3}$ buffer layer helped somewhat to decrease the surface roughness. On the other hand, annealing successfully reduced the roughness of the films to those of films grown with a lower power (figure 5).

In this study, all the AFM surface topography images were in very good agreement with the SEM surface morphology images (figure 3), although different locations were scanned over the surface. In addition, the columnar growth $\left(T_{\mathrm{d}} \geqslant 0.2-\right.$ $0.3 T_{\mathrm{m}}$ [35],) of the Ni films was observed from the crosssectional SEM images. The films with approximately $100 \mathrm{~nm}$ thick Ni layers had a higher as-grown roughness when deposited with substrate heating at $450{ }^{\circ} \mathrm{C}$ ( $\mathrm{Ni}$ films 9 and 10). Nevertheless, when we annealed these samples, roughness RMS values very close to those of the buffered films ( $\mathrm{Ni7}$ and 8 ) were achieved (figure 5). 


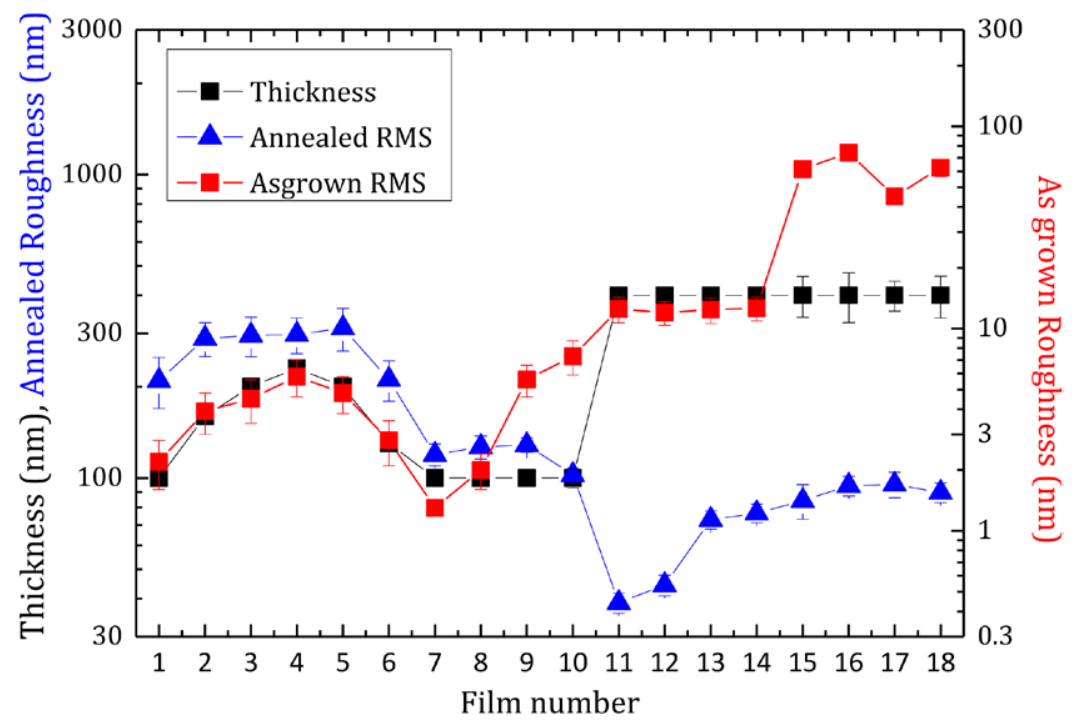

Figure 5. The relation between the thickness and surface roughness (RMS) values of all the as-grown and annealed at $900{ }^{\circ} \mathrm{C}$ cases. The as-grown roughness is drawn at a different scale to help visual comparison.

\subsection{Raman spectroscopy}

Graphene growth and characterization were carried out on the aforementioned eighteen $\mathrm{Ni}$ thin films using various CVD growth parameters. The growths were carried out in an atmospheric pressure CVD system (see online supporting information) (stacks.iop.org/JPhysD/48/455302/mmedia). Confocal Raman spectroscopy was used to assess the presence and the quality of the graphene. There are three prominent peaks in the Raman spectra of graphene: The $\mathrm{G}$ band, which is the only first order Raman scattering appearing at about $1585 \mathrm{~cm}^{-1}$; the $\mathrm{D}$ band appearing at about $1350 \mathrm{~cm}^{-1}$, which is induced by disorder; and the $\mathrm{G}^{\prime}$ band (also called $2 \mathrm{D}$ ) at about $2700 \mathrm{~cm}^{-1}$, achieved using laser excitation at $2.41 \mathrm{eV}$ (514nm wavelength). The number of graphene layers can be obtained from Raman measurements by comparing G-peak to $\mathrm{G}^{\prime}$-peak intensity $\left(I_{\mathrm{G}} / I_{\mathrm{G}}\right)$ and full width at the half maximum (FWHM) value of the $\mathrm{G}^{\prime}$ band (2D band) [36-38]. A large number of spectra were collected over a sample surface by manual focusing. Raman spectra were fit with Lorentzian functions to obtain peak position and FWHMs. In addition to the quality of fit (value of the coefficient of determination, or $R^{2}$ ) and FWHMs of the fit, the intensity ratios of the $\mathrm{G}$ and $\mathrm{G}^{\prime}$ bands $\left(I_{\mathrm{G}} / I_{\mathrm{G}}\right)$ were also compared. A single-peak Lorentzian fit to the $\mathrm{G}^{\prime}$ peak and $I_{\mathrm{G}} / I_{\mathrm{G}^{\prime}}<0.5$ indicates that the measurement is coming from mono-layer graphene and a two-peak Lorentzian fit points to bi-layer turbostratic graphene on the Ni film surface [39, 40].

A representative Raman spectrum of graphene growth (GRP20, graphene growth no. 20) on Ni1 film is shown in figure 6. For this measurement the $\mathrm{G}^{\prime}$ band can be fit with a Lorentzian peak with a FWHM of $28.9 \mathrm{~cm}^{-1}\left(R^{2}=0.9982\right)$. The same peak can also be fit by two Lorentzian peaks, but with FWHMs of $24.2 \mathrm{~cm}^{-1}$ and $24.5 \mathrm{~cm}^{-1}\left(R^{2}=0.9998\right)$. For the GRP20 sample, the $I_{\mathrm{G}} / I_{\mathrm{G}}$ ratio was about 0.30 and the FWHM of the $\mathrm{G}^{\prime}$ band was $29 \mathrm{~cm}^{-1}$. Although the Raman spectrum shows high-quality graphene, this kind of spectra was only available in sporadic areas of the sample. This is very probably due to the dewetting of the Ni film. When we increased the $\mathrm{CH}_{4}$ flow, the FWHMs of grown graphene layers decreased to a value of approximately $28 \mathrm{~cm}^{-1}$ with $I_{\mathrm{G}} / I_{\mathrm{G}}$ ratio of about 0.34 . Considering interlayer coupling and reported Raman measurements, these spectra may be assumed due to monolayer graphene $[2,38]$.

Among all the observed $\mathrm{G}^{\prime}$ band Raman spectra, when the number of graphene layers was more than two, the FWHM values were greater than $50 \mathrm{~cm}^{-1}$ and the $I_{\mathrm{G}} / I_{\mathrm{G}}$ ratio was larger than 1 . For these, the $\mathrm{G}^{\prime}$ band was fitted by more than three Lorentzian peaks. On the other hand, for 1 to 2-layer graphene, with a FWHM $<50 \mathrm{~cm}^{-1}$ and $I_{\mathrm{G}} / I_{\mathrm{G}^{\prime}}<1$, the $\mathrm{G}^{\prime}$ band can be fitted by at most three Lorentzian peaks.

\section{7. $\mathrm{CH}_{4}$ flow}

To enlarge the grain sizes in polycrystalline Ni films and thus to obtain larger graphene flakes, growth temperatures of 900 and $950{ }^{\circ} \mathrm{C}$ were used. At these temperatures, we found that there was a certain minimum requirement on the $\mathrm{CH}_{4}$ flow rate of $8 \mathrm{sccm}$, which led to the growth of 1-2 layer graphene with narrow $\mathrm{G}^{\prime}$ band FWHMs and with small D peaks. Moreover, 1-2-layer graphene flakes were sparsely distributed over thinner films $(\sim 100 \mathrm{~nm})$ at $1-8 \mathrm{sccm} \mathrm{CH}_{4}$ flow at $900{ }^{\circ} \mathrm{C}$ (an example of growth over Ni1 is given above). On the contrary, on thicker films (thickness $\geqslant 160 \mathrm{~nm}$ ), 1-2 layers of graphene grew with $8 \mathrm{sccm} \mathrm{CH}_{4}$ flow; however, their $\mathrm{G}^{\prime}$ peaks were broad with FWHMs of $38-56 \mathrm{~cm}^{-1}$ and the D and $\mathrm{D}^{\prime}$ peak intensities were larger compared to those obtained on thinner films (figure 7). Therefore, the $\mathrm{CH}_{4}$ flow was increased to 20-30 sccm for target $400 \mathrm{~nm}$ film thickness. Under a sufficiently high $\mathrm{CH}_{4}$ flow, the growth temperature of $900{ }^{\circ} \mathrm{C}$ was enough to considerably suppress the $\mathrm{D}$ and $\mathrm{D}^{\prime}$ intensity ratios. However, the $\mathrm{D}^{\prime}$ peak, which is also related to defects, became noticeable around $1610 \mathrm{~cm}^{-1}$ for these growths (an example, GRP50, is given in figure 7).

$\mathrm{Ni}$ films with buffer and/or adhesive layers (Ni11 and Ni12) had lower roughness RMS values after annealing. This led to the successful growth of 1-2-layer graphene on these 

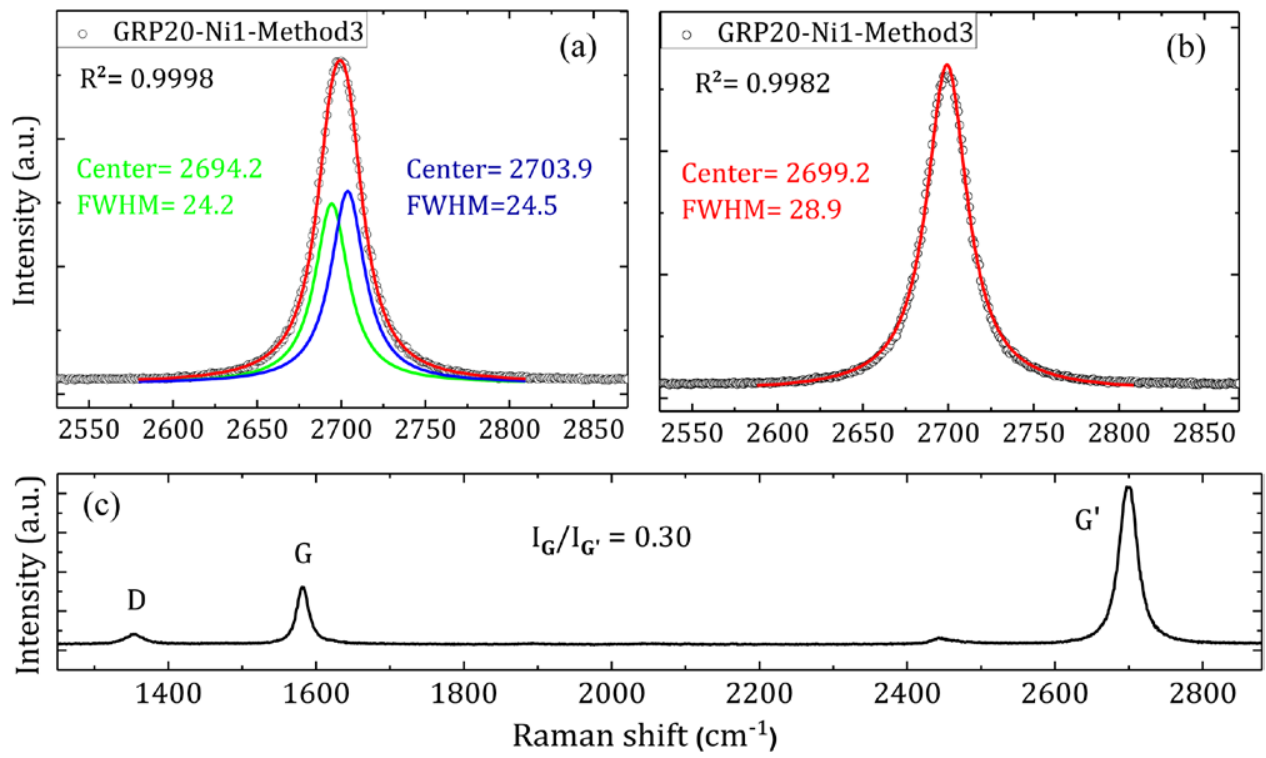

Figure 6. (a) $\mathrm{G}^{\prime}$ band Raman spectrum of a GRP20 sample grown on Ni1 film by method 3 (see the online supporting information, table S1) (stacks.iop.org/JPhysD/48/455302/mmedia). The green and blue lines are the components of the red line, which is the fitting result. (b) The same result fit with a single Lorentzian peak. (c) The whole range of the respective spectrum.

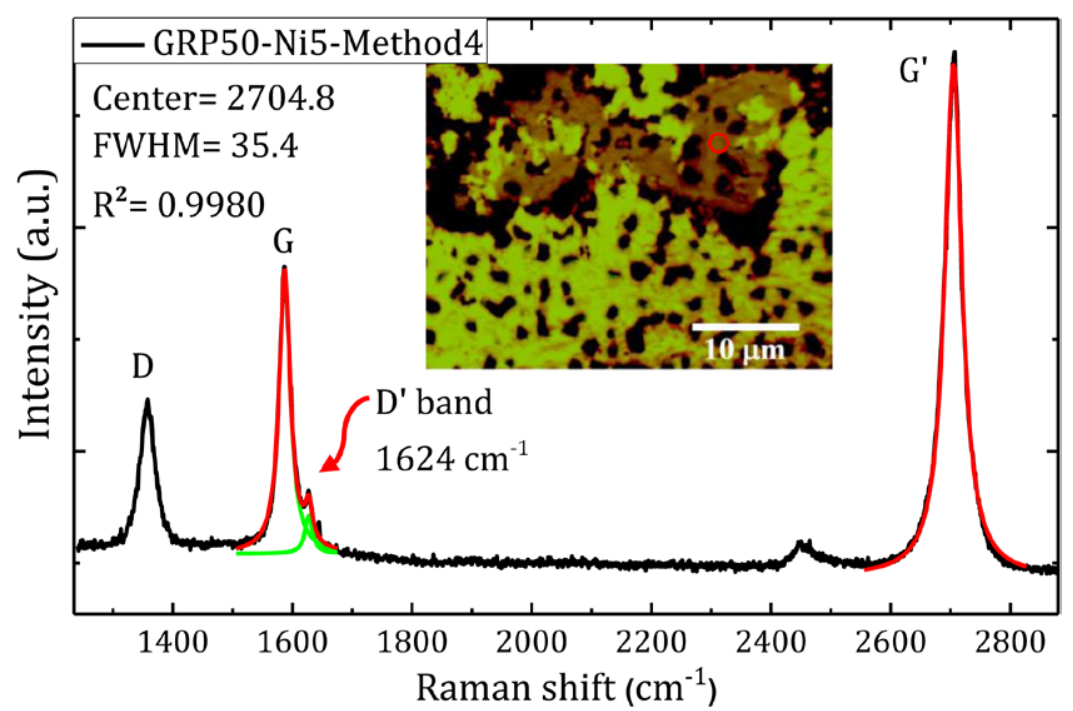

Figure 7. Raman spectrum of a GRP50 sample grown on a Ni5 substrate by method 4 (see the online supporting information, table S1) (stacks.iop.org/JPhysD/48/455302/mmedia). The green lines refer to the component of the red line, which is the calculated profile. The inset is the optical image of the area where the spectrum was collected and the red circle indicates the approximate position of the laser spot.

films. The large number of spectra collected over the surface by manual focusing confirms that a large portion of the surfaces were in fact covered by mostly 1-2 layer graphene.

\subsection{Graphene coverage}

Graphene surface coverage with the film thickness and buffer layer was also studied. The SEM images of graphene growth (GRP23) carried over Ni4 film at $900{ }^{\circ} \mathrm{C}$ are shown in figures 8 (a) and (b). $\mathrm{Ni} 4$ had a $\mathrm{Ni}$ film grown on $\mathrm{SiO}_{2} / \mathrm{Si}$ with no buffer layer and had the largest $(\sim 230 \mathrm{~nm}) \mathrm{Ni}$ thickness of the group. The annealed sample was extensively dewetted (see figure 3). An SEM image with graphene growth was taken at least the dewetted part of the sample (figures 8(a) and (b)). The darkest areas are the exposed $\mathrm{SiO}_{2}$ substrate. Graphene growth over the remaining Ni part can be seen as darker regions. The grain boundary regions can also be distinguished. GRP31 was grown on Ni14 film and GRP28 was grown on Ni11 film with the same growth conditions of GRP23. Ni14 film ( 400 nm) is thicker than Ni4 film, but also without a buffer layer, thus Ni film is less affected by dewetting. The effect of thickness on graphene coverage can be easily seen by comparing figures 8(a) and (b). Figure 8(c) has almost full coverage over the surface. Graphene grew over the Ni grains, yielding monolayer to multilayer growth. The line profile of SEM image, as indicated by a red line in figure 8(c), can be utilized to gain an impression about the number of graphene layers (figure 8(d)). This is in good agreement with the number of layers of graphene obtained by Raman spectroscopy. Comparing the sample grown over no buffer layer (figure 8(e)) and a buffer 
(a)

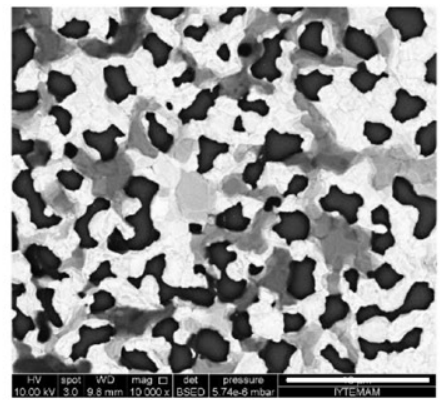

(c)

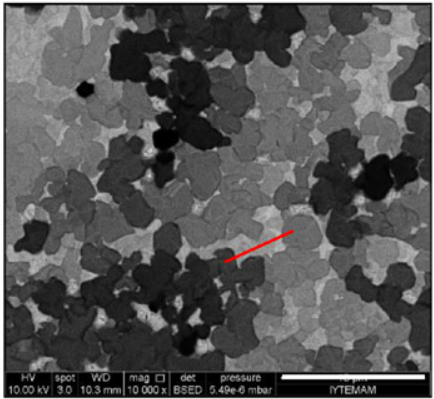

(e)

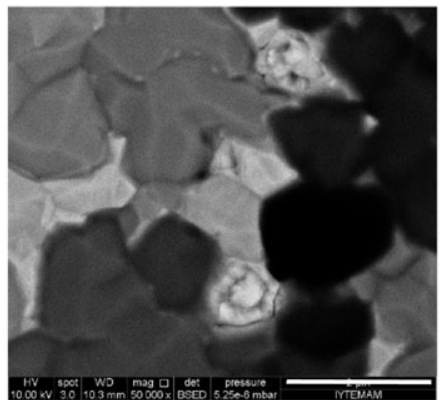

(b)

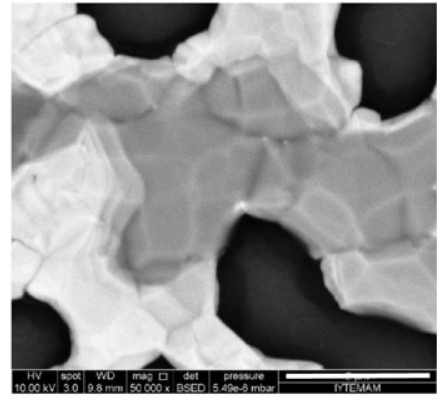

(d)

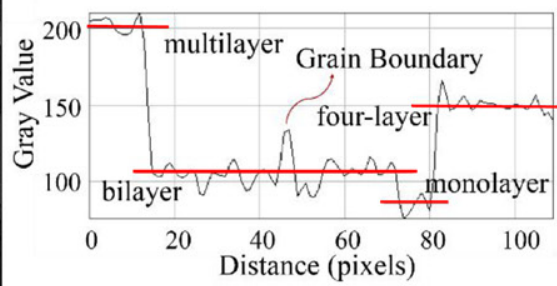

(f)

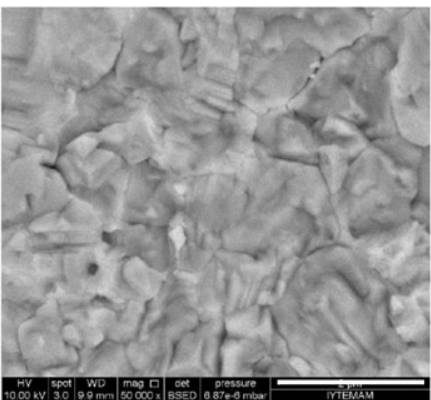

(g)

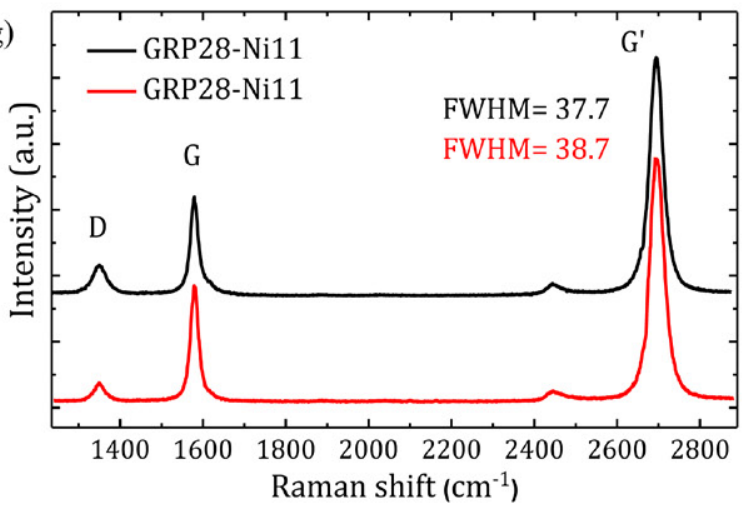

Figure 8. SEM images of GRP23 (a and b) GRP31 (c) and (e) and GRP28 (f) at different magnifications. (d) One-dimensional grey value analysis of the red line on (c). (g) Raman spectra of the GRP28 sample which was grown on the Ni11 substrate. The red spectrum and black spectrum belong to different areas on the same film surface. The scale bars on the SEM images of (b), (e), and (f) are $2 \mu \mathrm{m}$; (a) and (c) are $10 \mu \mathrm{m}$

layer (figure $8(f)$ ) of the same thickness and the same growth parameters clearly demonstrates the effect of the buffer layer. The film with a buffer layer had a continuous 1-2-layer graphene growth over the same range.

\section{Conclusions}

Our study indicated that for thinner films $(<230 \mathrm{~nm})$ the surface roughness increased with thickness for both the as-grown and annealed samples. On the other hand, for the annealed thicker films $(\sim 400 \mathrm{~nm})$, the surface roughness was inversely proportional to the thickness.

Increasing the annealing temperature resulted in preferential texture in the (111) orientation and increased the average grain size; however, it also increased dewetting. Including an $\mathrm{Al}_{2} \mathrm{O}_{3}$ buffer layer reduced the dewetting effect, a result that was more pronounced in thicker Ni films. Ni films with a thinner $\mathrm{Al}_{2} \mathrm{O}_{3}$ buffer layer (about $30 \mathrm{~nm}$ ) also had a more pronounced (111) orientated surface than non-buffered $\mathrm{Ni}$ films. Between films deposited with an $\mathrm{Al}_{2} \mathrm{O}_{3}$ buffer layer, a $\mathrm{Cr}$ adhesive layer, and both $\mathrm{Al}_{2} \mathrm{O}_{3} / \mathrm{Cr}$ layers, the effect of the 
$\mathrm{Cr}$ adhesive layer was greater than that of the $\mathrm{Al}_{2} \mathrm{O}_{3}$ buffer layer on texturing in the (111) orientation of the crystal. Film deposition at a substrate temperature of $450{ }^{\circ} \mathrm{C}$ led to (200) oriented $\mathrm{Ni}$ film growth. A $\mathrm{Cr}$ adhesive layer and $60 \mathrm{~min}$ annealing led to Ni films mostly textured in the (111) direction, even with the substrate heating during deposition. $\mathrm{Cr}$ layers increased the as-grown film surface roughness dramatically, but annealing restored the roughness to that of the same thickness films without an $\mathrm{Al}_{2} \mathrm{O}_{3}$ layer.

No graphene growth was observed at $800{ }^{\circ} \mathrm{C}$. At 900 and $950{ }^{\circ} \mathrm{C}$, a $\mathrm{CH}_{4}$ flow rate of $8 \mathrm{sccm}$ was necessary to grow mono or bi-layer graphene with narrow FWHMs and with small D peaks. Under a low $\mathrm{CH}_{4}$ flow rate over $\mathrm{Ni}$ film thicker than $160 \mathrm{~nm}$, mono or bi-layer graphene with broad $\mathrm{G}^{\prime}$ peak FWHMs $\left(38-56 \mathrm{~cm}^{-1}\right)$ and significant $\mathrm{D}$ and $\mathrm{D}^{\prime}$ peaks was obtained, whereas a $\mathrm{CH}_{4}$ flow rate of 20-30 sccm for $400 \mathrm{~nm}$ thick Ni films restored these values. Ni films with buffer and/ or adhesive layers had lower roughness RMS values after annealing and larger area graphene was successfully grown.

In summary, we suggest that growing high-quality, largearea, 1- or 2-layer graphene on polycrystalline Ni transition metal thin film is optimized by using buffer and/or adhesive layers.

\section{Acknowledgments}

This work was supported by TUBITAK (The Scientific and Technical Research Council of Turkey) with project number 112T946. We would like to thank Dr Bülent Aslan, Dr Uğur Serincan, and Güven Korkmaz for the majority of the XRD measurements. We also thank Dr Orhan Öztürk and Mehmet Fidan for the opportunity to perform some of the XRD measurements. Additionally, thanks to the IYTE Material Research Center staff.

\section{References}

[1] Yu Q, Lian J, Siriponglert S, Li H, Chen Y P and Pei S-S 2008 Graphene segregated on Ni surfaces and transferred to insulators Appl. Phys. Lett. 93113103

[2] Losurdo M, Giangregorio M M, Capezzuto P and Bruno G 2011 Graphene CVD growth on copper and nickel: role of hydrogen in kinetics and structure Phys. Chem. Chem. Phys. 13 20836-43

[3] Reina A, Thiele S, Jia X, Bhaviripudi S, Dresselhaus M S, Schaefer J A and Kong J 2009 Growth of large-area singleand bi-layer graphene by controlled carbon precipitation on polycrystalline Ni surfaces Nano Res. 2 509-16

[4] Li X, Cai W, Colombo L and Ruoff R S 2009 Evolution of graphene growth on $\mathrm{Ni}$ and $\mathrm{Cu}$ by carbon isotope labeling Nano Lett. 9 4268-72

[5] Grüneis A, Kummer K and Vyalikh D V 2009 Dynamics of graphene growth on a metal surface: a time-dependent photoemission study New J. Phys. 11073050

[6] Dedkov Y S and Fonin M 2010 Electronic and magnetic properties of the graphene-ferromagnet interface $\mathrm{New} \mathrm{J}$. Phys. 12125004

[7] Bertoni G, Calmels L, Altibelli A and Serin V 2005 Firstprinciples calculation of the electronic structure and EELS spectra at the graphene/Ni (111) interface Phys. Rev. B 71075402

[8] Zhang Y, Gomez L, Ishikawa F N, Madaria A, Ryu K, Wang C, Badmaev A and Zhou C 2010 Comparison of graphene growth on single-crystalline and polycrystalline $\mathrm{Ni}$ by chemical vapor deposition J. Phys. Chem. Lett. $13101-7$

[9] Zhao W, Kozlov S M, Höfert O, Gotterbarm K, Lorenz M P A, Viñes F, Papp C, Görling A and Steinrück H-P 2011 Graphene on $\mathrm{Ni}(111)$ : coexistence of different surface structures J. Phys. Chem. Lett. 2 759-64

[10] Carel R, Thompson C V and Frost H J 1996 Computer simulation of strain energy effects vs surface and interface energy effects on grain growth in thin films Acta Mater. 44 2479-94

[11] Thiele S, Reina A, Healey P, Kedzierski J, Wyatt P, Hsu P-L, Keast C, Schaefer J and Kong J 2010 Engineering polycrystalline Ni films to improve thickness uniformity of the chemical-vapor-deposition-grown graphene films Nanotechnology 21015601

[12] Wintterlin J and Bocquet M-L 2009 Graphene on metal surfaces Surf. Sci. 603 1841-52

[13] Baraton L, He Z B, Lee C S, Cojocaru C S, Châtelet M, Maurice J-L, Lee Y H and Pribat D 2011 On the mechanisms of precipitation of graphene on nickel thin films Europhys. Lett. 9646003

[14] Shelton J C, Patil H R and Blakely J M 1974 Equilibrium segregation of carbon to a nickel (111) surface: a surface phase transition Surf. Sci. 43 493-520

[15] Obraztsov A N, Obraztsova E A, Tyurnina A V and Zolotukhin A A 2007 Chemical vapor deposition of thin graphite films of nanometer thickness Carbon 45 2017-21

[16] Lee G-H, Cooper R C, An S J, Lee S, van der Zande A, Petrone N, Hammerberg A G, Lee C, Crawford B and Oliver W 2013 High-strength chemical-vapor-deposited graphene and grain boundaries Science 340 1073-6

[17] Grantab R, Shenoy V B and Ruoff R S 2010 Anomalous strength characteristics of tilt grain boundaries in graphene Science 330 946-8

[18] Li X et al 2009 Large-area synthesis of high-quality and uniform graphene films on copper foils Science 324 1312-4

[19] O'Brien M and Nichols B 2010 CVD synthesis and characterization of graphene thin films Army Research Laboratory ARL-TR-5047 www.arl.army.mil/www/default. cfm?technical_report $=1915$

[20] Thompson C V 1990 Grain growth in thin films Annu. Rev. Mater. Sci. 20 245-68

[21] Petersen J and Mayr S 2008 Dewetting of Ni and NiAg solid thin films and formation of nanowires on ripple patterned substrates J. Appl. Phys. $\mathbf{1 0 3} 023520$

[22] Luber E J, Olsen B C, Ophus C and Mitlin D 2010 Solid-state dewetting mechanisms of ultrathin Ni films revealed by combining in situ time resolved differential reflectometry monitoring and atomic force microscopy Phys. Rev. B 82085407

[23] Gong Y, Zhang X, Liu G, Wu L, Geng X, Long M, Cao X, Guo Y, Li W and Xu J 2012 Layer-controlled and wafer-scale synthesis of uniform and high-quality graphene films on a polycrystalline nickel catalyst Adv. Funct. Mater. 22 3153-9

[24] Noda S, Hasegawa K, Sugime H, Kakehi K, Zhang Z, Maruyama S and Yamaguchi Y 2007 Millimeter-thick single-walled carbon nanotube forests: hidden role of catalyst support Japan. J. Appl. Phys. 46 L399

[25] Dikonimos Makris T, Giorgi L, Giorgi R, Lisi N and Salernitano E 2005 CNT growth on alumina supported nickel catalyst by thermal CVD Diamond Relat. Mater. 14 815-9

[26] Hata K, Futaba D N, Mizuno K, Namai T, Yumura M and Iijima S 2004 Water-assisted highly efficient synthesis 
of impurity-free single-walled carbon nanotubes Science 306 1362-4

[27] Zhu X, Cheng D and Kuai P 2008 Catalytic decomposition of methane over $\mathrm{Ni} / \mathrm{Al}_{2} \mathrm{O}_{3}$ catalysts: effect of plasma treatment on carbon formation Energy Fuels 22 1480-4

[28] Cassell A M, Raymakers J A, Kong J and Dai H 1999 Large scale CVD synthesis of single-walled carbon nanotubes J. Phys. Chem. B 103 6484-92

[29] Teo K B K, Chhowalla M, Amaratunga G A J, Milne W I, Hasko D G, Pirio G, Legagneux P, Wyczisk F and Pribat D 2001 Uniform patterned growth of carbon nanotubes without surface carbon Appl. Phys. Lett. 79 1534-6

[30] Kir S 2009 Growth and electrical characterization of high purity carbon nanotubes Master Thesis Izmir Institute of Technology, İzmir

[31] Saiz E, Cannon R M and Tomsia A P 2008 High-temperature wetting and the work of adhesion in metal/oxide systems Annu. Rev. Mater. Res. 38 197-226

[32] Chae S J, Güneş F, Kim K K, Kim E S, Han G H, Kim S M, Shin H J, Yoon S M, Choi J Y and Park M H 2009 Synthesis of large-area graphene layers on poly-nickel substrate by chemical vapor deposition: wrinkle formation Adv. Mater. 21 2328-33

[33] Butt M A, Chughtai A, Ahmad J, Ahmad R, Majeed U and Khan I 2008 Theory of adhesion and its practical implications J. Fac. Eng. Technol. 2007 21-45

[34] Fogarassy Z, Rümmeli M H, Gorantla S, Bachmatiuk A, Dobrik G, Kamarás K, Biró L P, Havancsák K and
Lábár J L 2014 Dominantly epitaxial growth of graphene on Ni (1 111$)$ substrate Appl. Surf. Sci. 314 490-9

[35] Thompson C V and Carel R 1996 Stress and grain growth in thin films J. Mech. Phys. Solids 44 657-73

[36] Reina Ceeco A 2010 Single-and few-layer graphene by ambient pressure chemical vapor deposition on nickel Doctor of Philosophy Thesis Massachusetts Institute of Technology, Cambridge

[37] Reina A, Jia X, Ho J, Nezich D, Son H, Bulovic V, Dresselhaus M S and Kong J 2008 Large area, few-layer graphene films on arbitrary substrates by chemical vapor deposition Nano Lett. 9 30-5

[38] Gomez de Arco L M 2010 Graphene and carbon nanotubes: synthesis, characterization and applications for beyond silicon electronics Doctor of Philosophy Thesis University of Southern California, Los Angeles

[39] Shibuta Y and Elliott J A 2011 Interaction between two graphene sheets with a turbostratic orientational relationship Chem. Phys. Lett. 512 146-50

[40] Hwang J S, Lin Y H, Hwang J Y, Chang R, Chattopadhyay S, Chen C J, Chen P, Chiang H P, Tsai T R and Chen L C 2013 Imaging layer number and stacking order through formulating Raman fingerprints obtained from hexagonal single crystals of few layer graphene Nanotechnology 24015702 